\title{
Hydrodynamic forces acting on an oscillating structure
}

\author{
D. Mirauda ${ }^{1}$, A. Volpe Plantamura ${ }^{1} \& \mathrm{~S}$. Malavasi $^{2}$ \\ ${ }^{1}$ D.I.F.A. Basilicata University, Italy \\ ${ }^{2}$ D.I.I.A.R. Politecnico di Milano, Italy
}

\begin{abstract}
The submerged and semi-submerged structures (off-shore platforms, wind farms, floating buildings, marine turbines, oil conduits, buoys) in sea currents are often subjected to strong vibrations that condition the structural stability and also give rise to serious damage to the mechanical parts of the structures, with consequent efficiency loss of the system and above all, repercussions in the environment in which they are located. These vibrations increase when the structures are immersed in bounded flows (presence of the bed and/or the free surface). In order to attenuate and eliminate these problems, analytical and experimental means are used to describe the dynamic behaviour of structures for different flow field configurations and boundary conditions. Recent research has demonstrated that integrated numerical modelling (considering both structure and fluid characteristics) is the more suitable way to value the dynamic response of a system. To check on the reliability of the resolution methods experimental controls are needed that, for the complexity of structures, requires models with simplified geometries (spheres, cylinders, rectangles). In this context, the present work aims to provide some experimental results obtained analyzing both the oscillations and the forces acting on a structure to simple geometry (sphere) characterized by low values of mass and damping and invested by a free surface steady flow.
\end{abstract}

Keywords: sphere, free surface, bed, hydrodynamic forces.

\section{Introduction}

The study of flow-structure interactions is important in many scientific fields as in hydraulics, in civil and environmental engineering, in river, maritime and in 
ocean constructions. In particular, the analysis of the hydrodynamic forces and the evaluation of vibrate modes of structures in water are fundamental for the correct design of off-shore structures like wind farms, marine turbines, oil conduits and platforms, buoys, river constructions, bridges, embankments and also new plants producing electric energy by the sea currents.

The complex phenomena generating by the fluid-structure interaction depend by different elements: the physic characteristics of the fluid (density and dynamic viscosity), the flow cinematic conditions, the location of the structure respect to the depth of the current (level of submergence of the system in the current) and also the mechanical characteristics of the structure itself.

All these elements can cause conditions of structural instability, with consequent efficiency loss and repercussions in the sea environment.

At the moment, these problems are exceeded through an increasing of the offshore structures dimensions, assuring in this way high security coefficients to the detriment to a general reduction of costs, but above all amplifying the environmental impact, causing visual externalities, when the installation is not completely submerged and located near the coast.

In order to eliminate these problems, recent research (see Jonkman and Sclavounos [1]) have demonstrated as the integrated numerical modelling, considering the characteristics of both structure and fluid, is the more suitable way to value correctly the dynamic response of such systems and thus to give more information for a better design. The difficulty of this type of approach consists in the check on the reliability of the resolution methods. Because of the complexity of the structures, these checks require models with simplified geometries (spheres, cylinders, rectangles, etc.). For this purpose, it is important to underline as the study of the dynamic response of a structure at simple geometry is fundamental to make deeper the analysis of these complex phenomena. Moreover, simple geometries allow an easier comparison between the experimental and numerical approach which, also if with approximate solutions, gives certain advantages in the modelling of complex structures.

In literature there is a large number of fundamental studies on the fluidstructure interactions of submerged cylinders (two-dimensional bodies) (Sarpkaya [2], Griffin and Ramberg [3], Bearman [4], Parkinson [5], Williamson and Govardhan [6], Blevins [7], Naudascher and Rockwell [8], Sumer and Fredsøe [9], and Anagnostopoulos [10]), but there are fewer more recent studies on three-dimensional structures (spheres), because of the difficult interpretation and analysis of the flow fields generating around these bodies (Williamson and Govardhan [11]; Govardhan and Williamson [12, 13]; Jauvtis et al. [14], Mirauda and Greco [15, 16]; Franzetti et al. [17]; Greco et al. [18]; Mirauda et al. [19-21], and Volpe Plantamura et al. [22]).

In order to characterize the phenomena involving the interaction between fluid and three-dimensional structures, this paper analyzes in detailed way the response of a spherical body in a free surface steady flow to change of the location of the structure inside the current. The knowledge of the behaviour of the body in these conditions could be interesting because could avoid the structural over-dimensioning of the same. 


\section{Experimental details}

Experiments have been performed in a non-tilted Plexiglas open water channel in the Hydraulics Laboratory of the Politecnico di Milano, having a rectangular cross section $(0.6 \mathrm{~m}$ high, $0.5 \mathrm{~m}$ wide and $7.0 \mathrm{~m}$ long) just used and characterized in previous works (Malavasi and Guadagnini [23]). A water filled sphere, with a diameter $D=0.087 \mathrm{~m}$ and a mass $m=0.46 \mathrm{Kg}$, has been used as the obstacle. The sphere surface is made of PVC and covered with an episodic paint to reduce the surface roughness. A rod, made of stainless steel and Delrin, has been used to connect the sphere to a fixed structure mounted on the channel. The stainless steel part, which is connected to the sphere, is $0.080 \mathrm{~m}$ long and $0.003 \mathrm{~m}$ in diameter, while the Delrin part completing the rod has a diameter of $0.012 \mathrm{~m}$.

The sphere-rod system is sustained by a robust support realized with aluminium bars, fixed to the channel which permits at the sphere to move in the both directions (stream wise, $x$, and transversal respect to the current flow, $y$ ).

In all the experiments the depth of current was equal to 5 times the sphere diameter $(H=0.435 \mathrm{~m})$, in order to maintain the blockage coefficient constant $\left(\gamma_{b}=0.03\right.$, ratio between the body frontal area and the transverse section of the channel), while the mean flow velocity, $U$, changed between 0.2 and $0.5 \mathrm{~m} / \mathrm{s}$. The sphere, instead, was located in three different submergence conditions respect to the flow, $h^{*}$ (ratio between the distance from free surface and upper surface of the sphere and the body diameter): the first at an elevation of $2 D$ above the bottom wall of the channel and from the free surface, in order to assume the mimicking of the symmetrical flow condition for the sphere $\left(h^{*}=2\right)$ and to neglect the wall and free surface influence (fig. 1(a)); the second at a distance of $3 \mathrm{~mm}\left(h^{*}=3.97\right)$ from the bottom (fig. 1(b)), with the aim to value if the wall closeness have relevant effects on the dynamic response of the body; finally, near the free surface $\left(h^{*} \approx 0\right)$ in asymmetric bounded flow (fig. 1(c)), in order to analyze the effects of the free surface distortion on transverse and stream wise oscillation amplitudes.

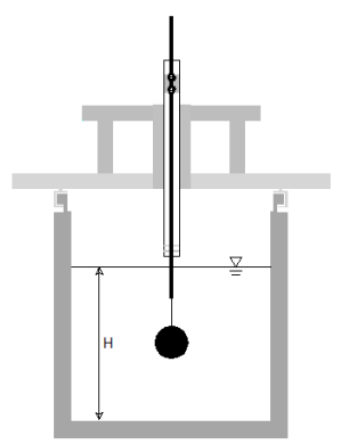

(a)

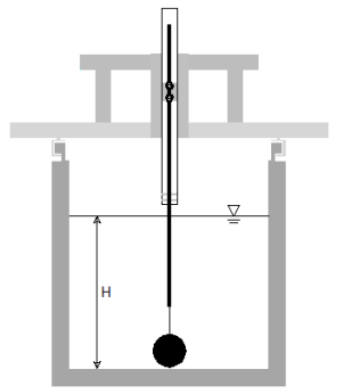

(b)

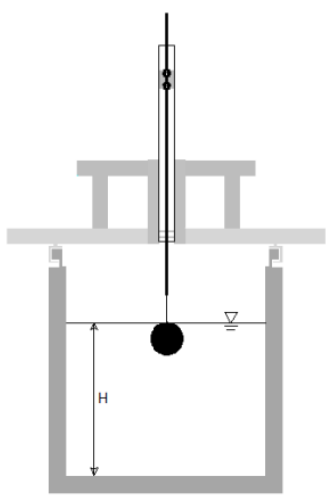

(c)

Figure 1: Transverse section of the channel with sphere at: (a) $h^{*}=2$; (b) $h^{*}=3.97$, (c) $h^{*}=0$. 
The sphere movements, for the different flow velocities $U$ (or likewise for the normalized velocity $U^{*}=U / f_{n w} D$, where $f_{n w}$ is the body natural frequency in water), have been measured by an image analysis through a CCD camera (Charge Coupled Device) set on acquisition frequencies from $30 \mathrm{~Hz}$ to $50 \mathrm{~Hz}$. The camera, located below the water channel, allows us to memorize the $2 \mathrm{D}$ displacements, thanks to the presence of a marker placed below the centre of the sphere in line with its centre of gravity. Table 1 summarizes the experimental tests of the present work.

Table 1: $\quad$ Experimental tests.

\begin{tabular}{|c|c|c|c|c|c|c|c|c|c|c|c|c|}
\hline TEST & $\mathbf{1}$ & $\mathbf{2}$ & $\mathbf{3}$ & $\mathbf{4}$ & $\mathbf{5}$ & $\mathbf{6}$ & $\mathbf{7}$ & $\mathbf{8}$ & $\mathbf{9}$ & $\mathbf{1 0}$ & $\mathbf{1 1}$ & $\mathbf{1 2}$ \\
\hline$h^{*}$ & 2 & 3.97 & 0 & 2 & 3.97 & 0 & 2 & 3.97 & 0 & 2 & 3.97 & 0 \\
\hline$U(\mathrm{~m} / \mathrm{s})$ & \multicolumn{3}{|c|}{0.2} & \multicolumn{3}{|c|}{0.3} & \multicolumn{3}{c|}{0.4} & \multicolumn{3}{c|}{0.5} \\
\hline$U^{*}$ & \multicolumn{3}{|c|}{1.90} & \multicolumn{3}{|c|}{2.84} & \multicolumn{3}{c|}{3.79} & \multicolumn{3}{|c|}{4.74} \\
\hline$R e$ & \multicolumn{2}{|c|}{17400} & 26100 & \multicolumn{3}{c|}{34800} & \multicolumn{3}{|c|}{43500} \\
\hline
\end{tabular}

\section{Analytical approach}

The forces acting on the sphere in the flow principal direction, $F_{x}$, and in transverse that, $F_{y}$, have been valued by means of the system of two uncoupled dynamic equilibrium equations:

$$
\begin{aligned}
& m \ddot{x}+c \dot{x}+k x=F_{x} \\
& m \ddot{y}+c \dot{y}+k y=F_{y}
\end{aligned}
$$

in which the instantaneous velocity $(\dot{x}, \dot{y})$ and acceleration $(\ddot{x}, \ddot{y})$ of the sphere have been obtained by a Centred Finite Difference Method (CFDM) with error of $4^{\text {th }}$ order for both the first and the second derivative, thanks to the knowledge of the displacements ( $x$ and $y$ ). Because the scheme of the equations of the used CFDM does not change for the two directions, we report below the system only for the stream wise case:

$$
\begin{aligned}
& \dot{x}_{i}=\frac{x_{i-2}-8 x_{i-1}+8 x_{i+1}-x_{i+2}}{12 \Delta T}+\frac{1}{30} x(\xi) \Delta T^{4} \\
& \ddot{x}_{i}=\frac{-x_{i-2}+16 x_{i-1}-30 x_{i}+16 x_{i+1}-x_{i+2}}{12 \Delta T^{2}}+\frac{1}{90} \cdots(\xi) \Delta T^{4}
\end{aligned}
$$

where $\Delta T$ represents the sampling time or the inverse of the acquisition frequency.

The numerical resolution of the eqn. (1) has required the knowledge of the modal parameters of spring constant, $k$, and damping coefficient, $c$. Such parameters have been calculated through the analysis of the free oscillations of the sphere in air (fig. 2). In particular, the spring constant has been derived by the expression of the natural frequency in air, valued by the power spectrum of the free oscillation (fig. 3):

$$
f_{n}=\frac{1}{2 \pi} \sqrt{\frac{k}{m}}
$$


The value obtained of the spring constant was $k=36.72 \mathrm{Kg} / \mathrm{s}^{2}$.

Instead, the damping parameter, $c$, has been deduced by the following equation:

$$
\zeta=\frac{1}{2 \pi} \sqrt{\frac{c}{k m}}
$$

in which the damping ratio, $\zeta$, has been valued through the classical method of logarithmic decrement applied to the successive displacement peaks of the same free oscillation signal shown in fig. 2. The value of the damping ratio was $\zeta=0.0044$, while the structural damping was $c=0.036 \mathrm{Kg} / \mathrm{s}$. Finally, the values of $f_{n w}$ and $\zeta_{w}$ in water equal to $1.21 \mathrm{~Hz}$ and 0.004 , have been obtained multiplying the eqns. (3) and (4) for the coefficient $\left[\mathrm{m}^{*} /\left(\mathrm{m}^{*}+C_{A}\right)\right]^{1 / 2}$, where $C_{A}$ is the added mass coefficient taking into account the water presence and is equal to 0.5 for spheres, while $m^{*}$ is the mass ratio equal to the ratio between the mass of the sphere and the displaced fluid mass $\left(m^{*}=m / \pi \rho D^{3} / 6=1.34\right)$.

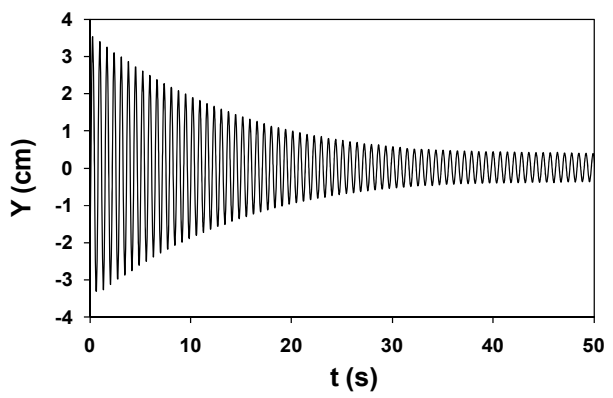

Figure 2: $\quad$ Evolution in the time of the free oscillation of the sphere.

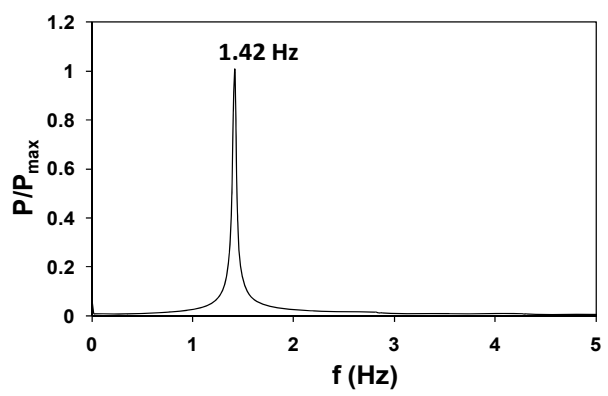

Figure 3: $\quad$ Power spectrum of the free oscillation of the sphere.

\section{Forces analysis}

In order to study the behaviour of the structure at the different positions inside the current, $h^{*}=2, h^{*}=3.97$ and $h^{*}=0$, the displacements in the flow principal direction and transverse to the current and the respective acting hydrodynamic forces have been evaluated. 
In particular the following figures report the stream wise and transverse oscillations normalized through the characteristic dimension of the body, that in this case is represented by the sphere diameter, for the lower $\left(U^{*}=1.90\right)$ and higher normalized velocity $\left(U^{*}=4.74\right)$ of the investigated currents and the relative forces normalized respect to the observed maximum value.

In the case of $h^{*}=2$, representing the more studied condition in literature, an increase of stream wise and transverse displacements is observed to increasing of the mean flow velocity (fig. 4(a)). In particular, the stream wise oscillations for $U^{*}=4.74$ increase of 15 times respect to the case of $U^{*}=1.90$, while the transverse those of around 75 times. The major increase of transverse displacements implies a trajectory at shape of crescent topology, observed also in literature for spheres with similar mass ratio and completely submerged. Obviously, the mean displacement of the sphere in the main flow direction respect to the static position (zero position) increases with the mean flow velocity. The increase of the displacements is confirmed by an increasing of the forces. In fact, the stream wise forces in fig. 4(b) show an increase of the mean component with the increasing of the velocity, while the fluctuating component seems to be lightly growing with $U^{*}$. On the contrary, in fig. 4(c), the transverse forces show a more
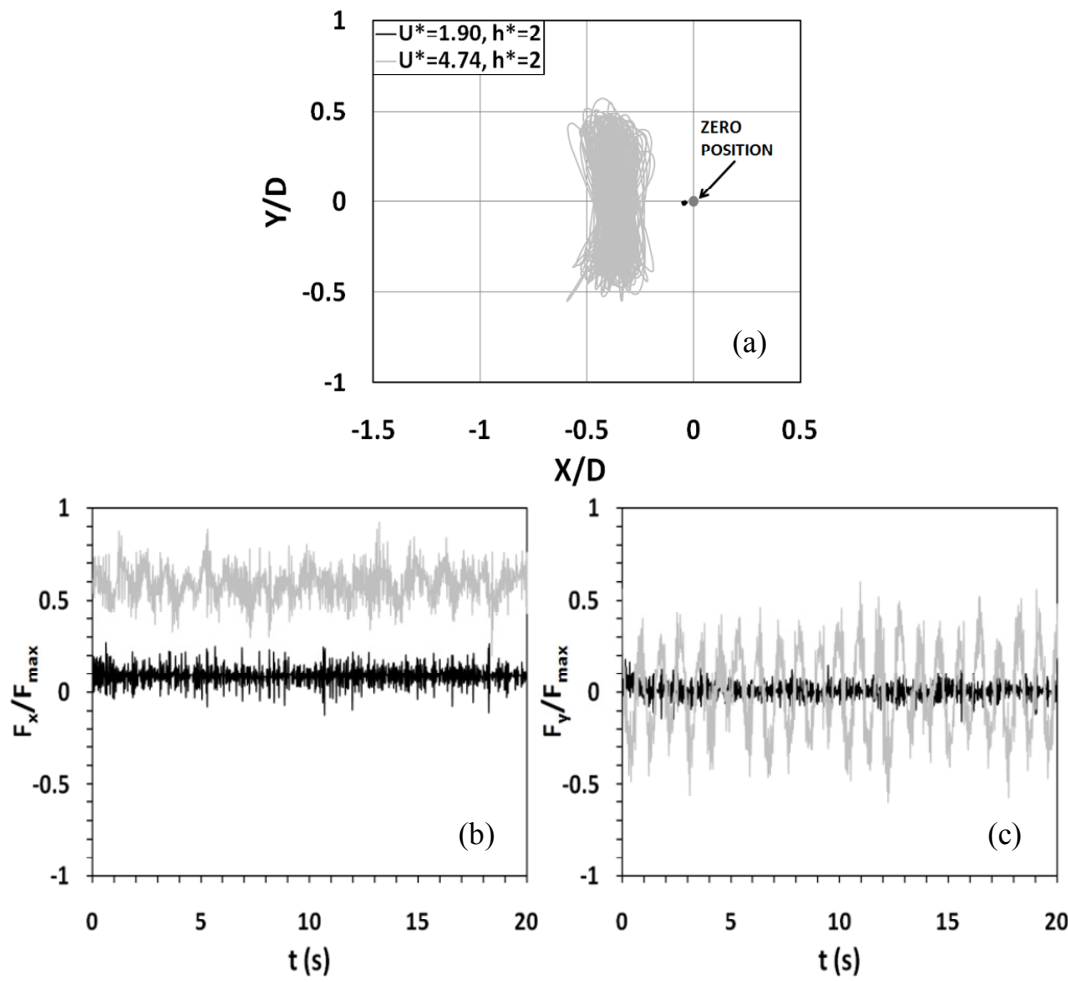

Figure 4: (a) Trajectory of the sphere at $h^{*}=2$ for $U^{*}=1.90$ and 4.74; (b) and (c) evolution in the time of stream wise $F_{x}$ and transverse forces $F_{y}$. 
significant increasing of the fluctuating component with the increasing of the velocity, while the mean component remains constant around the zero value for the body symmetry condition (fig. 4(c)).

Similar results to the case of $h^{*}=2$ can be observed for the sphere placed near the bottom of the channel, $h^{*}=3.97$ (fig. 5). In this case, the asymmetrical boundary condition due to the closeness of the wall lightly influences the sphere movements in respect to the previous case. In particular, as it is possible to note from fig. 5(a), the presence of the channel floor significantly reduces the stream wise and transverse mean displacements respecting the condition of $h^{*}=2$. For the higher velocity, $U^{*}=4.74$, we note a reduction of about $20 \%$ in stream wise and of about $10 \%$ in the transverse mean displacements. Also in this case the shape of the trajectory for high velocity is that at crescent topology. The slight differences in the displacements are however less evident in terms of mean and fluctuating values of the acting forces (fig. 5(b) and 5(c)).
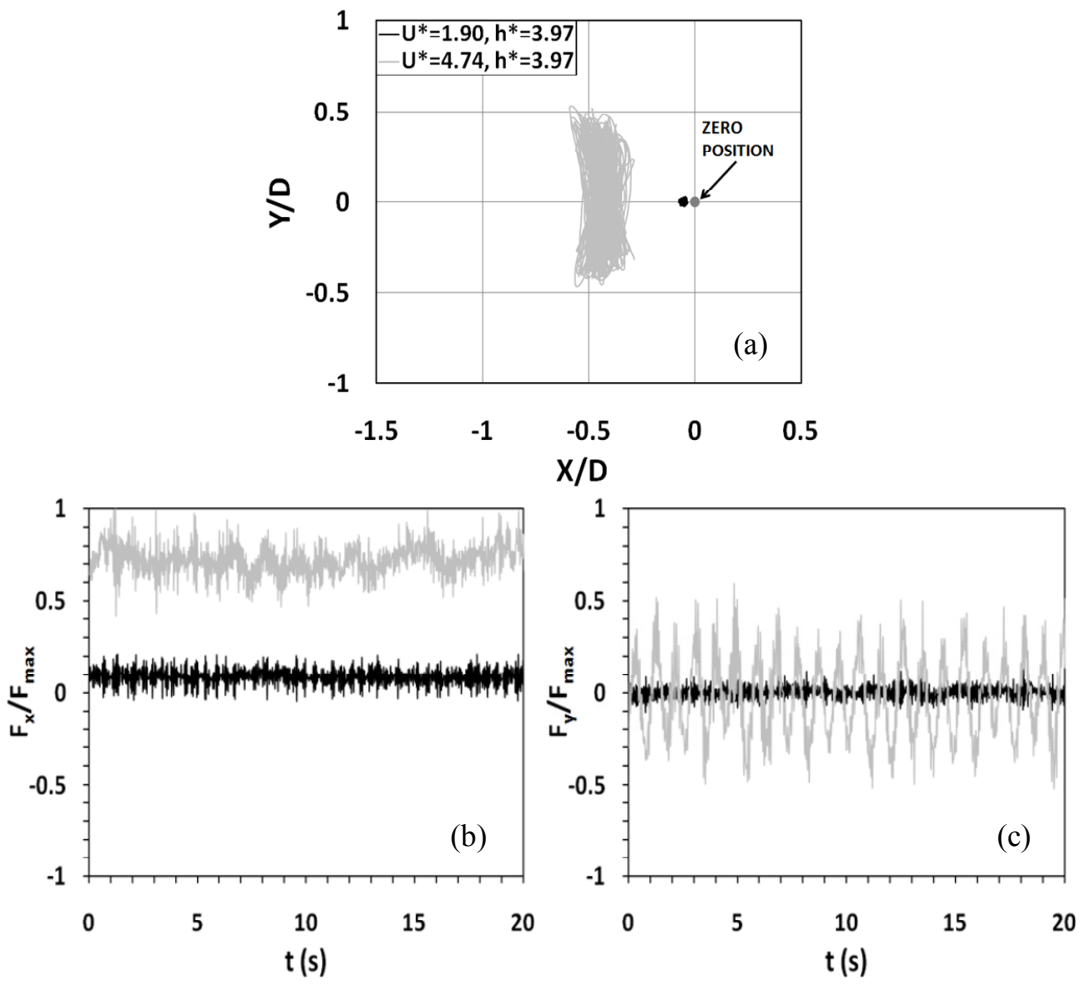

Figure 5: (a) Trajectory of the sphere at $h^{*}=3.97$ for $U^{*}=1.90$ and 4.74; (b) and (c) evolution in the time of stream wise $F_{x}$ and transverse forces $F_{y}$.

Comparing the results of $h^{*}=2$ and $h^{*}=3.97$ with the case of body close to the free surface $\left(h^{*}=0\right)$, we observe as the latter presents a very different behaviour 
both in terms of the sphere movements and acting forces. Fig. 6(a) shows as for the two different flow cinematic conditions the sphere trajectories are similar, and present stream wise and transverse displacements comparable. This experimental result confirms that reported by Mirauda et al. [21] and Volpe Plantamura et al. [22], putting in evidence displacements ranges around to values of about $0.1 D$, and showing a chaotic trajectory. This behaviour could be explained with the strong distortion of the free surface that reduces the transverse movements of the sphere for higher flow velocities and increases the body displacements in the both directions for lower normalized velocities. Instead, as observed in the previous cases, correctly at $U^{*}=4.74$ the sphere results more displaced in the principal flow direction respect to the static position. These results appear confirmed from the analysis of the forces acting on the structure. Fig. 6(b) shows as the mean value of the stream wise force, $F_{x}$, for $U^{*}=4.74$ is translated less towards the high respect to the other cases of relative submergence, and the fluctuations are less growing with the increase of the current velocity. From the fig. 6(c) it is possible to note as, for the two analyzed conditions of current flow velocity, also in this case, the mean value of $F_{y}$ is
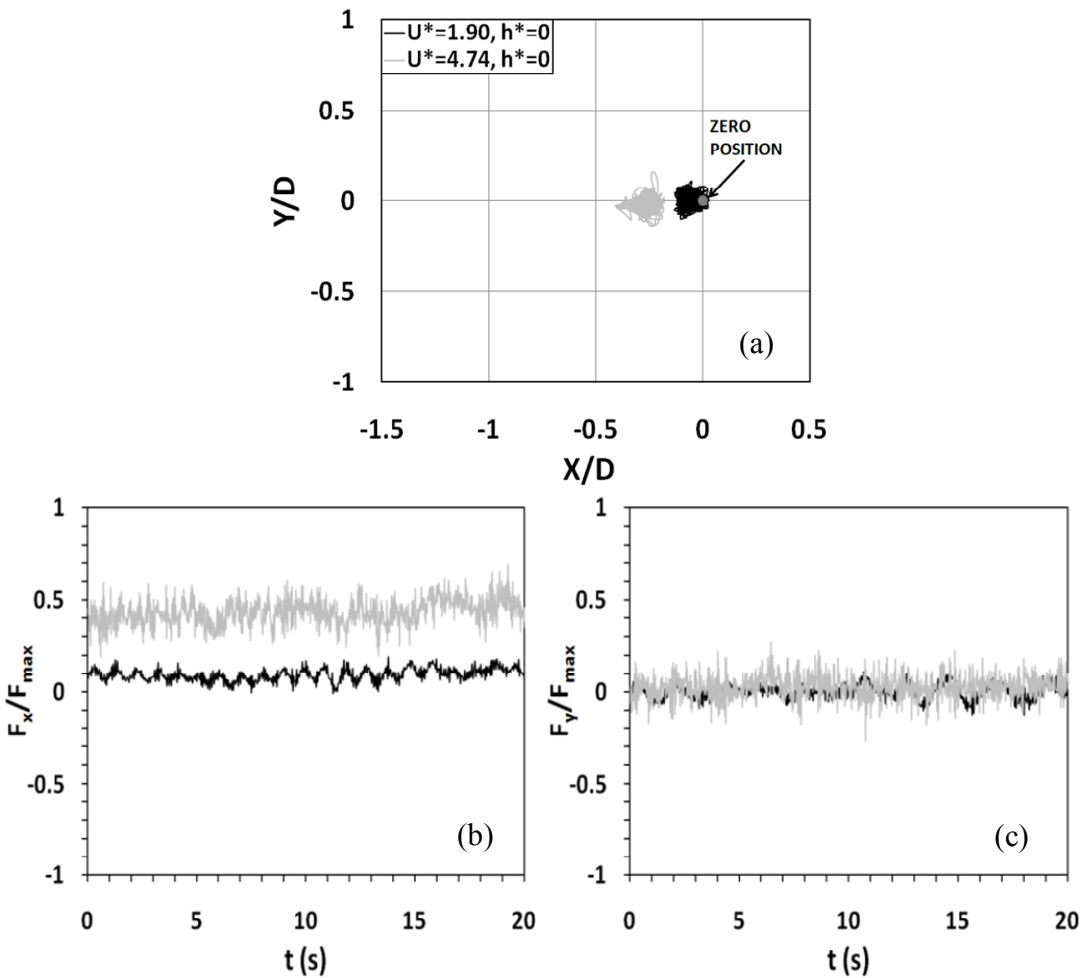

Figure 6: (a) Trajectory of the sphere at $h^{*}=0$ for $U^{*}=1.90$ and 4.74 ; (b) and (c) evolution in the time of stream wise $F_{x}$ and transverse forces $F_{y}$. 
correctly equal to zero because of the symmetry of the sphere, while, instead, in different way respect to the other submergence conditions, the force fluctuations respect to the mean value for $U^{*}=4.74$ are strongly reduced.

Fig. 7 summarizes and compares the behaviour of the cinematic and dynamic parameters characteristic of the structure response for the two different conditions of normalized velocity $\left(U^{*}=1.90\right.$ and 4.74) and for the three considered relative submergences $\left(h^{*}=2,3.97\right.$ and 0$)$. In particular, the root mean square values of stream wise and transverse components of normalized displacements and forces are reported. In the stream wise direction, it is evident as the displacements and forces values are always crescent with the current velocity; while for the transverse direction it is shown clearly the different trend of the fluctuating values of both displacements and forces, when the sphere is close to the free surface at $h^{*}=0$.

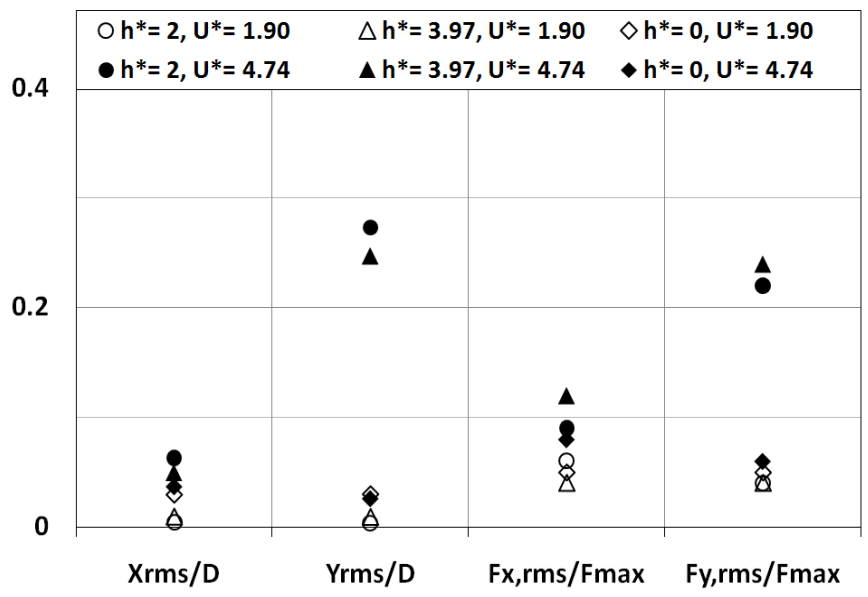

Figure 7: Root mean square values of normalized displacements and forces in stream wise and transverse direction, for $h^{*}=2,3.97$ and 0 at low $\left(U^{*}=1.90\right)$ and high normalized velocity $\left(U^{*}=4.74\right)$.

\section{Conclusions}

In the present work we have analyzed the forces acting on a spherical structure characterized by a low mass-damping parameter $m^{*} \zeta=0.005$ and immersed in a steady flow at constant blockage coefficient and at three different relative submergences: two asymmetrical bounded condition, with sphere close to the free surface $\left(h^{*}=0\right)$ and to the bottom $\left(h^{*}=3.97\right)$ and a symmetrical position $\left(h^{*}=2\right)$, which represents the more analyzed condition in literature.

The results evidence the strong influence of the closeness of the free surface $\left(h^{*}=0\right)$ both on the sphere movements and acting hydrodynamic forces, respect to the symmetrical condition of complete submergence $\left(h^{*}=2\right)$ and to the asymmetrical condition to the bottom $\left(h^{*}=3.97\right)$. In particular, the free surface 
distortion induces two different effects: a reduction of displacements and force fluctuations in the transverse direction for high current flow velocity, $U^{*}=4.74$, and an increase of stream wise and transverse movements and forces, even if not much significant, in the case of low normalized velocity, $U^{*}=1.90$.

\section{Acknowledgements}

The authors express their sincere thanks to Dr. Luca Martinelli, of the Department of Structural Engineering, Politecnico di Milano, for his precious assistance in the choice and resolution of the analytical method for the evaluation of the forces acting on the body.

\section{References}

[1] Jonkman, J.M. \& Sclavounos, P.D., Development of Fully Coupled Aeroelastic and Hydrodynamic Models for Offshore Wind Turbines: Preprint. ASME Wind Energy Symposium, Reno, Nevada, January 10-12, 2006.

[2] Sarpkaya, T., Vortex-induced oscillations. ASME J. Appl. Mech., 46, pp. 241-258, 1979.

[3] Griffin, O.M. \& Ramberg, S.E., Some recent studies of vortex shedding with application to marine tubulars and risers. ASME Journal of Energy Resour. Tech., 104, pp. 2-13, 1982.

[4] Bearman, P. W., Vortex shedding from oscillating bluff bodies. Annual Review of Fluid Mechanics, 16, pp. 195-222, 1984.

[5] Parkinson, G., Phenomena and modelling of flow-induced vibrations of bluff bodies. Prog. In Aerospace Sci., 26, pp. 169-224, 1989.

[6] Williamson, C. H. K. \& Govardhan, R., Vortex-induced vibrations. Annual Review of Fluid Mechanics, 36, pp. 413-455, 2004.

[7] Blevins, R. D., Flow-Induced Vibrations. Van Nostrand Reinhold, 1990.

[8] Naudascher, E. \& Rockwell, R., Flow-Induced Vibrations: An Engineering Guide. Balkema, 1994.

[9] Sumer, B. M. \& Fredsøe, J., Hydrodynamics around Cylindrical Structures. World Scientific, 1997.

[10] Anagnostopoulos, P., Flow-Induced Vibrations in Engineering Practice. WIT Press, 2002.

[11] Williamson, C. H. K. \& Govardhan, R., Dynamics and forcing of a tethered sphere in a fluid flow. Journal of Fluids and Structures, 11, pp. 293-305, 1997.

[12] Govardhan, R. \& Williamson, C. H. K., Vortex induced motions of a tethered sphere. Journal of Wind Engineering Industrial Aerodynamics, 6971, pp. 375-385, 1997.

[13] Govardhan, R. \& Williamson, C. H. K., Vortex-induced vibrations of a sphere. Journal of Fluid Mechanics, 531, pp. 11-47, 2005. 
[14] Jauvtis, N., Govardhan, R. \& Williamson, C. H. K., Multiple modes of vortex-induced vibration of a sphere. Journal of Fluids and Structures, 15, pp. 555-563, 2001.

[15] Mirauda, D. \& Greco, M., Transverse vibrations of an sphere at high combined mass-damping parameter. In Shallow Flows, eds. Jirka \& Uijttewaal, Balkema Publisher, Taylor \& Francis Group, London, pp. 111115, 2003.

[16] Mirauda, D \& Greco, M., Flow-induced vibration of an elastically sphere at high combined mass-damping parameter. Journal of IASME Transactions, 1, pp. 486-491, 2004.

[17] Franzetti, S., Greco, M., Malavasi, S. \& Mirauda, D., Flow Induced Excitation on basic shape structures. In Vorticity and turbulence effects in fluid structure interaction, eds. Brocchini M., Trivellato F., Collana AFM Advances in Fluid Mechanics, Wessex Institute of Technology Press, Southampton, United Kingdom, pp. 131-156, 2005.

[18] Greco, M., Malavasi, S., \& Mirauda, D., Interaction between a tethered sphere and a free surface flow. In Fluid Structure Interaction and Moving Boundary Problems, eds. Chakrabarti S.K., Hernandez S., Brebbia C.A., Wit PRESS, Southampton, Boston, 84, pp. 205-213, 2005.

[19] Mirauda, D., Malavasi, S., Greco, M. \& Volpe Plantamura, A., Kinematic analysis of the movement of a tethered sphere immersed in a free surface flow. In 9th International Conference on Fluid Control, Measurement and Visualization, Flucome 2007, Tallahassee, Florida, USA, September 16-19, Paper 151, 2007.

[20] Mirauda, D., Malavasi, S. \& Volpe Plantamura, A., Analysis of effects of the free surface on the movement of a tethered sphere immersed in a steady flow. In 9th International conference on flow-induced vibrations, FIV 2008, eds.: Zolotarev I., Horacek J., Praga, 30 June - 3 July, pp. 835-840, 2008.

[21] Mirauda, D., Volpe Plantamura A., Malavasi, S. \& Martinelli, L., Forze idrodinamiche agenti su una struttura oscillante. In XXXII Convegno di Idraulica e Costruzioni Idrauliche, ed. Walter Farina, Palermo, Italy, September 14-17, 2010.

[22] Volpe Plantamura, A., Mirauda, D. \& Malavasi, S., Analisi dell'influenza della superficie libera sulle vibrazioni di una sfera investita da una corrente stazionaria ed uniforme. L'Acqua, 4, pp. 71-76, 2010.

[23] Malavasi, S. \& Guadagnini, A., Interactions between a rectangular cylinder and a free surface flow. Journal of Fluids and Structures, 23, pp. 11371148, 2007. 